\title{
Hybrid Model for PAPR Minimization in OFDM System
}

\author{
Mohammed A. Hussein ${ }^{1}$, Ala'a.H.Ali², Ali K. Nahar ${ }^{3}$ \\ Electrical Engineering Dept, University of Technology, Baghdad, Iraq. \\ 316417@student.uotechnology.edu.iq, 140007@uotechnology.edu.iq, 30081@uotechnology.edu.iq.
}

\begin{abstract}
The technology has been growing rapidly in the form of portable wireless devices that can perform multiple functions to cope with the state-of-the-art technology and synchronization. A total device capacity must be increased to accommodate new wireless applications. This can be achieved by leveraging new technologies, with higher data rates. Spectrum pooling has gained immense popularity, with increased demand for frequency range and bandwidth availability constraints. Statistics suggest that much of the spectrum licensed is not used all the time. Because of the transmitter's nonlinearity nature, the large (peak to average power ratio (PAPR)) phenomenon is a drawback in orthogonal frequency division multiplexing (OFDM). Several hybrid approaches have recently been implemented to minimize PAPR's high value, at the expense of increasing the level of computational complexity in the system. In this paper, a new hybrid approach has been introduced in parallel to combine the selective mapping approach (SLM) with the partial transmit sequence (PTS) approach to improve the efficiency of PAPR reduction with lower numerical method complexity. The findings reveal that the OFDM systems with the proposed hybrid approach have better efficiency in terms of PAPR elimination, side-information, and computational complexity compared to current hybrid methods. Also, a hybrid approach proposed output could be maintained without degradation.
\end{abstract}

Index Terms: OFDM, PTS, PAPR, SLM

\section{INTRODUCTION}

Today fourth-generation cellular networking networks have been used in several countries around the world. Also, there are some challenges, such as an explosion of wireless mobile applications and services that cannot even be addressed by (4G), such as spectrum scarcity and higher energy use [1]. Fourth-generation communications are based on (Orthogonal Frequency Multiplexing Division (OFDM)) [2]. The OFDM is used in single-frequency networks such as digital audio broadcasting (DAB), digital video broadcasting (DVB), and indoor wireless systems such as IEEE802.15 [3], IEEE 802.11 [4], IEEE802.16 [5], and 4G-LTE [6]. A special situation is OFDM transmission with multiple carriers. By adding guard bands, this divides a bandwidth into two subcarriers and isolates them. Such carriers overlap, yet due to the presence in the form of the pulse, they are orthogonal. Using the Cyclic Prefix (CP), Inter-Symbol Interference (ISI) can be eliminated. OFDM offers advantages such as fading resistance, easier channel equalization, security, interference, and efficiency in computing. But the good side lobes and the strict timing criteria make bandwidth performance a sadness. 
On the other hand, other strategies have been suggested to reduce the high PAPR pattern in the OFDM method, including coding techniques [7], clipping and filtering techniques [8], constellation extension techniques [9], nonlinear compounding transformation schemes [10], and interleaving techniques [11], multiple signal representation (MSR) such as selective level mapping (SLM)[12], the partial transmission scheme (PTS)[13] .The MSR strategies are an appealing alternative because of their excellent efficiency, which can be applied without degradation of the signal [14]. The conventional PTS (C-PTS) scheme between the aforementioned techniques is a well-known deterministic non-signal distortion approach that delivers excellent PAPR reduction efficiency, but is set to increase the difficulty of measurement and involves side details.

SLM and Interleaving are considered to be another multiple signal representation (MSR) technique schemes to provide a lower computational difficulty, The degradation cost in PAPR performance reduction [15]. Several experiments have been conducted in recent years to merge the two forms of MSR approaches and use each method's inherent feature in one deterministic way.

Several methods were presented in the literature to combine PTS with SLM to reduce PAPR-value. By adding the input data sequence to the SLM technique and then forwarding each changed sequence to the PTS technique, Pushkarev [16] implemented a hybrid algorithm focused on the combination of SLM and PTS. Pushkarev method can have stronger PAPR reduction efficiency than C-PTS and SLM strategies, although the computational difficulty is greater than that of the C-PTS system.

K. Singh [17] and A. Mohammed [18] Combined the SLM and PTS approaches with a different method by adding SLM to the input data series and choosing the resulting OFDM signal as the PTS stream. The Singh's approach will accomplish PAPR reduction efficiency better than C-PTS and SLM schemes as the similarity between sub-carriers inside the sub-blocks are reduced, but the level of computational complexity and side knowledge is reduced compared with the C-PTS technique.

Tiwari proposed another tactic in [19] by mixing the SLM and PTS schemes. The method of Tiwari depended first on the application of the SLM technique and then the resulting OFDM signal was transferred to the FFT block before the C-PTS technique was implemented. The Tiwari's method is superior to the PAPR reduction C-PTS process, to the disadvantage of the growing machine and side knowledge sophistication.

Also, Xiaoqiang [20] introduced an algorithm that depends on the combination of interleaving and PTS techniques in which the permutation data sequence corresponding to the optimum time-domain sequence is transferred to the C-PTS technique to further improve PAP efficiency. Xiaoqiang algorithm PAPR reduction efficiency is better than CPTS. Mathematical formulas and bits of side information, however, are far larger than the C-PTS method. 
In this paper, the suggested hybrid scheme (SLM-CSS-PTS) is implemented, where several factors have been measured to develop a realistic, excellent PAPR reduction approach, such as PAPR reduction efficiency, computational complexity, side details, Bit error rate output and Power Spectral Density (PSD). Simulation results and numerical analyses show that the proposed hybrid scheme has better performance compared to current hybrid schemes in terms of PAPR reduction, low computational complexity and low side details. Additionally, the hybrid approach proposed preserves the BER and PSD efficiency without degradation.

\section{PAPR BASED ON OFDM}

In practical applications, the high PAPR value in multi-carrier systems based on orthogonal transmissions, such as OFDM, is classified as the major disadvantage these systems face. The ratio of the maximum transmitted signal peak power divided by the mean signal power can be defined as PAPR [22]. Fig.1 displays a condensed OFDM device diagram in which one of the modulation families maps the input data sequence first, and then parallel shift to the IFFT node. IFFT modifies the baseband input sequence by the orthogonality of the subcarriers and transforms the frequency domain output sequence to the time domain. The cyclic prefix (CP) is applied to the signal before transmission, such that the OFDM signal may be interpreted as

$$
x(n)=\frac{1}{\sqrt{N}} \sum_{k=0}^{N-1} X(k) e^{j 2 \pi k \frac{n}{N}} \quad, \quad 0 \leq n \leq N-1
$$

where $X(k)$ the input data sequence, and $(N)$ is the sub-carrier numbers. On the receiver side also, all transmitter operations performed are reversed. In OFDM system, the signal output consists of the summation as a sinusoidal form of several different modulated subcarriers. Because of the design of the IFFT array, the instantaneous peak strength of certain sinusoid signal subcarriers may be combined to become far greater than the average signal capacity; providing such subcarrier phases are identical [23]. PAPR in decibel $(\mathrm{dB})$ could be formulated in mathematical terms as [24]

$$
\operatorname{PAPR}(\mathrm{dB})=10 \log _{10} \frac{\max |x(n)|^{2}}{E\left\{|x(n)|^{2}\right\}},
$$

where $E\{$.$\} represents the mean value of the signal. Furthermore, a popular method for determining$ PAPR output is the CCDF (Complementary Cumulative Distribution Function) [25]. The CCDF based on PAPR denotes the probability that the PAPR value exceeds a certain OFDM symbol threshold (PAPR0)

$$
\operatorname{Pr}\left(\mathrm{PAPR}>P A P R_{0}\right)=1-\left(1-\exp \left(-P A P R_{0}\right)\right)^{N L},
$$


(L) is Oversampling Factor. The OFDM baseband signal continuous-time (nearly) could be achieved by implementing L-time over-sampling of the distinct OFDM signal to collect those signal peaks that do not occur while measuring the PAPR value. The oversampling process is done by adding (L-1) N zeros between the subcarriers. The sampling of the discrete baseband signal with $L>4$ is also necessary to improve the precision of the PAPR value [26].

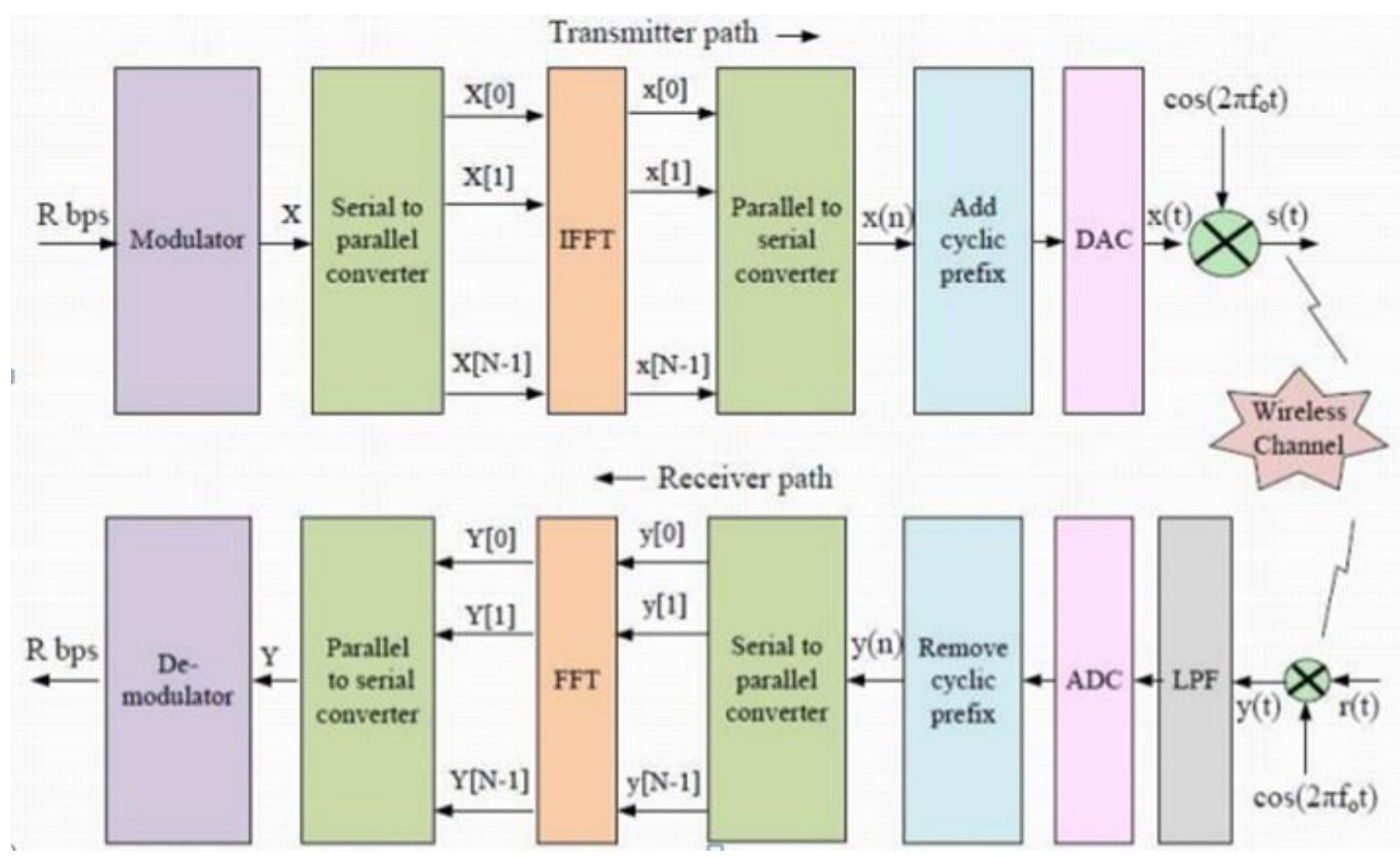

FIG. 1: BLOCK DIAGRAM FOR OFDM [23].

\section{PAPR REDUCTION TECHNIQUES}

PAPR reduction involves several methods and this depends on varying factors like reduction capability, increased transmission signal strength, computational complexity, data rate loss, peak reduction carrier, BER increase, spectral efficiency. PAPR reduction methods were suggested to minimize the high PAPR [27] as often as practicable. Several of these methods: clipped and filtered, Tone Injection, Partial Transmit Sequence, coding, compounding, Tone Reservation, Selected Mapping (SLM), etc.

Two techniques will be discussed in this articles.:

\section{A. Partial Transmit Sequence (PTS):}

Muller introduced the partial transmission sequence technique in 1997 to improve the performance of PAPR reduction in the OFDM system [28]. In Fig.2, Partial Transmission Sequence (PTS) is a undistortion system that divides the input information block into several disorganized subs-blocks and executes IFFT. Such sub-block outputs of the IFFT are scrambled or weighted by a set of rotational determinants that are summed up to create specific candidate signals. In the end, the lowest PAPR signal for transmission is chosen [29]. As long as a PTS has to be applied, the uncertainty that is a significant 
parameter in the transmitter must be taken into account because it increases exponentially with the quantity of the subsequence. For this purpose, the rotation of the vector should be limited to a set with a finite number of elements PTS, which is hindered by a high computational complexity [30] resulting from a thorough search of candidate signals and additionally allows data concerning rotation factors to be sent as side information to the receiver.

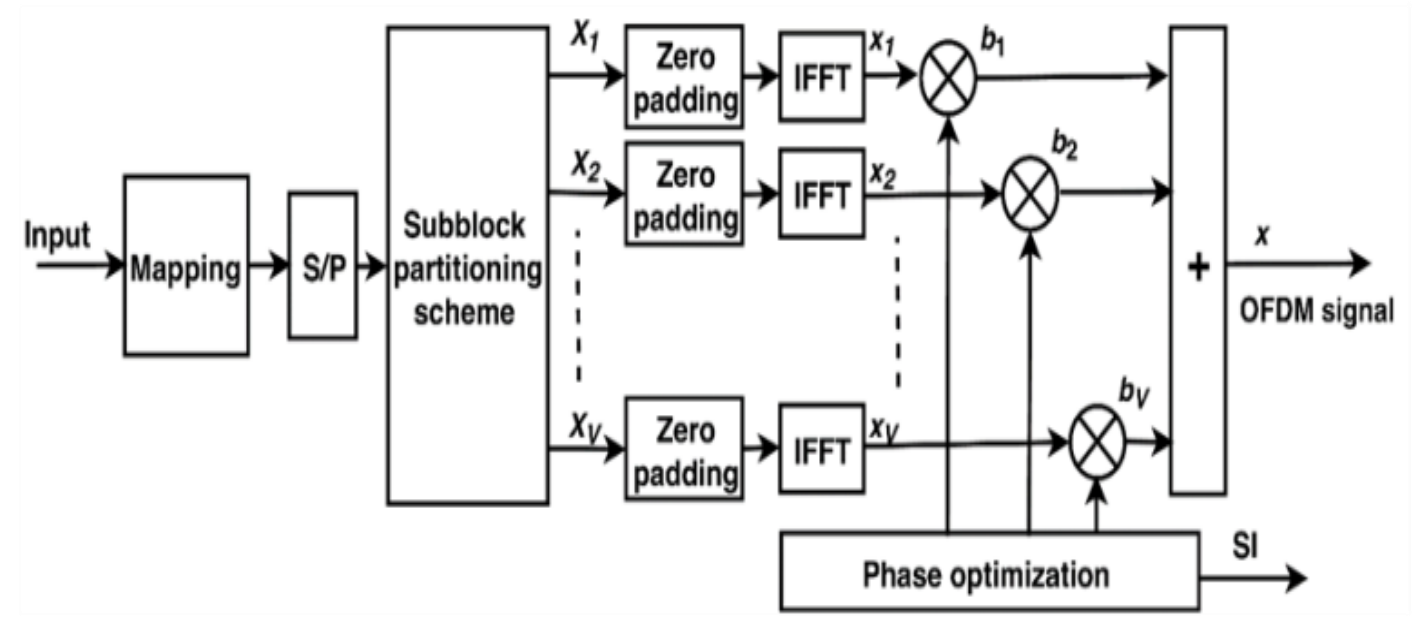

FIG. 2: PTS BLOCK DIAGRAM.

The advantage of PTS: has low complexity and Introduces less distortion.

Drawbacks of PTS: BER performance degrades, side information is required and involve complex transmitter quantities of the vector.

\section{B. Selected Mapping (SLM):}

In 1995, Bauml suggested the selective mapping technique to reduce the value of PAPR within the OFDM system [31]. SLM 's simple concept is to create alternate U transmission sequences seeded from the same data source and then pick the transmitted signal with the lowest PAPR. The idea stems from the assumption that as the PAPR is calculated from the sequence of data vectors transmitted, $X^{u}$ can alter the PAPR properties after the IFFTT by multiplying the data vectors by some random step. A set of $U$ markedly distinct, pseudo random fixed vectors is generated

$$
P^{u}=\left[P_{u, 0}, P_{u, 1}, \cdots, P_{u, N-1}\right], \text { where }(u)=[1,2, \ldots, U]
$$

mathematically,

The data, $\mathrm{X}^{\mathrm{u}}$, are multiplied subcarrier wise with each one of the $\mathrm{U}$ vectors, $\mathrm{P}^{(\mathrm{u})}$, resulting in a set of $\mathrm{U}$ different possible transmit symbols, $\mathrm{X}^{(\mathrm{u})}$ as depicted in

$X^{u}=\left[X_{0} P_{u, 0}, X_{1} P_{u, 1}, \cdots, X_{N-1} P_{u, N-1}\right]$ 
Next, all $\mathrm{U}$ possible transmit vectors are transferred to the to the time domain via the IFFT, $\mathrm{x}$ ${ }^{(\mathrm{u})} \mathrm{m}=\mathrm{IFFT}\left\{\mathrm{X}^{(\mathrm{u})}\right\}$, and the transmit symbol with the lowest PAPR, $\mathrm{X}^{\mathrm{U}}$, is chosen for transmission. A SLM transmitter block structure is depicted in Fig 3.

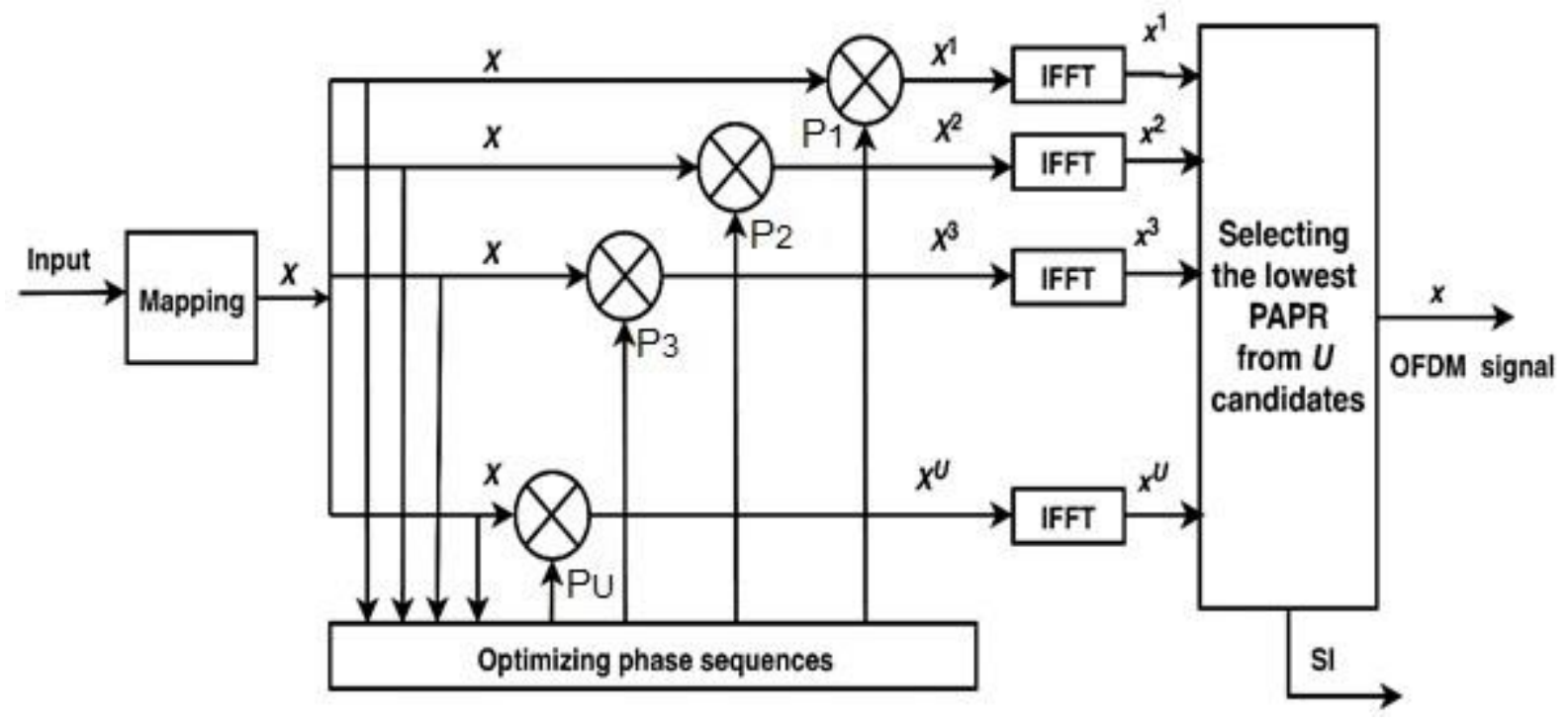

FIG. 3. SLM BLOCK DIAGRAM

Side information is also an essential problem with SLM as it is appropriate to notify the recipient which vector $\mathrm{P}^{(\mathrm{u})}$ was used. $\log _{2}(\mathrm{U})$ bits are required to send this data to increase redundancy. Since the loss of this information (in a fading channel) means that the full lack of transmit symbol channel coding is necessary, redundancy is further enhanced in order to ensure proper data recovery at the receiver.

The advantage of SLM is: Number of carriers separated, it does not introduce any distortion, while the disadvantages of SLM are: Degrades efficiency at BER, there is a need for side data.

\section{PROPOSED METHOD}

A new approach to combine the SLM method and the CSS-PTS method is proposed, so it can be termed the SLM-CSS-PTS method. This method aims to reduce the PAPR value and the complexity of the computation better than the C-PTS technique. The procedure for reducing the PAPR value in the SLM method is to generate $(\mathrm{U})$ phase rotation vectors $(\mathrm{PRV}), P^{u}=\left[P_{u, 0}, P_{u, 1}, \cdots, P_{u, N-1}\right]$, where $(u)=$ $[1,2, \ldots, U]$, and $(N)$ is the length of the data sequence [34]. Moreover, the original data sequence, $X=\left[X_{0}, X_{1}, \cdots, X_{N-1}\right]$, is copied according to a combined number of PRVs [33]. Next, U-PRVs multiply the data sequence component-wise to generate the new independent sequences [34],

$$
X^{u}=\left[X_{0} P_{u, 0}, X_{1} P_{u, 1}, \cdots, X_{N-1} P_{u, N-1}\right] .
$$

After that, the independent sequences are transferred to the IFFT bank to produce a range of candidate signals, then the candidate signal that achieves the lowest PAPR value is selected with the optimal PRV index for transmission [21].

$$
x^{u}=\operatorname{IFFT}\left(X^{u}\right) \quad, \quad u=1,2, \cdots, U .
$$


In the CSS-PTS method, the procedure for reducing the PAPR value starts after obtaining the time-domain sub-block sequences $\left\{x_{v} \mid v=1,2, \ldots, V\right\}$, in which the $g_{\text {th }}$ candidate sequence, $1 \leq g \leq G$, is generated by cyclically shifted some sub-block sequences and combined them [35]. The OFDM signal can thus be written as

$$
x=\sum_{v=1}^{V} x_{v}^{g}, 1 \leq g \leq G,
$$

where $x_{v}^{g}$ is the cyclically shifting version of $x_{v}$ by some of the integer shift numbers? This is [36],

$$
\begin{aligned}
x_{v}^{g}=\operatorname{circular}\left(x_{v}, q_{v}^{g}\right)=[ & x_{v}\left(q_{v}^{g}\right), x_{v}\left(q_{v}^{g}+1\right), \ldots, x_{v}(N+1), x_{v}(0), \\
& \left.\ldots, x_{v}\left(q_{v}^{g}-1\right)\right]
\end{aligned}
$$

where $q_{v}^{g}, 1 \leq v \leq V$, is the shift number. Also, the set of shift numbers in the $g_{\text {th }}$ candidate sequence is denoted by $H^{g}=\left[q_{1}^{g}, q_{2}^{g}, \ldots, q_{V}^{g}\right]$. Hence, the CSS-PTS method needs to construct G-shift number sets, $H=\left[H^{1}, H^{2}, \ldots, H^{G}\right]$, To generate the OFDM signals needed for the candidate [32]. Among the candidate sequences, the candidate with the minimum PAPR is selected with the correct optimum shift number for transmission to the receiver. In the (SLM-CSS-PTS) method, the reduction protocol for PAPR is to combine the SLM method with the CSS-PTS method in parallel, where each method is used to process half the input data sequences and both halves are again combined before transmitting to the receiver. Fig.4 shows the SLM-CSS-PTS method, where the sequence of input data is divided into two equal parts $\mathrm{A}$ and $\mathrm{B}$,

$$
X=[X \mathrm{~A}, X \mathrm{~B}],
$$

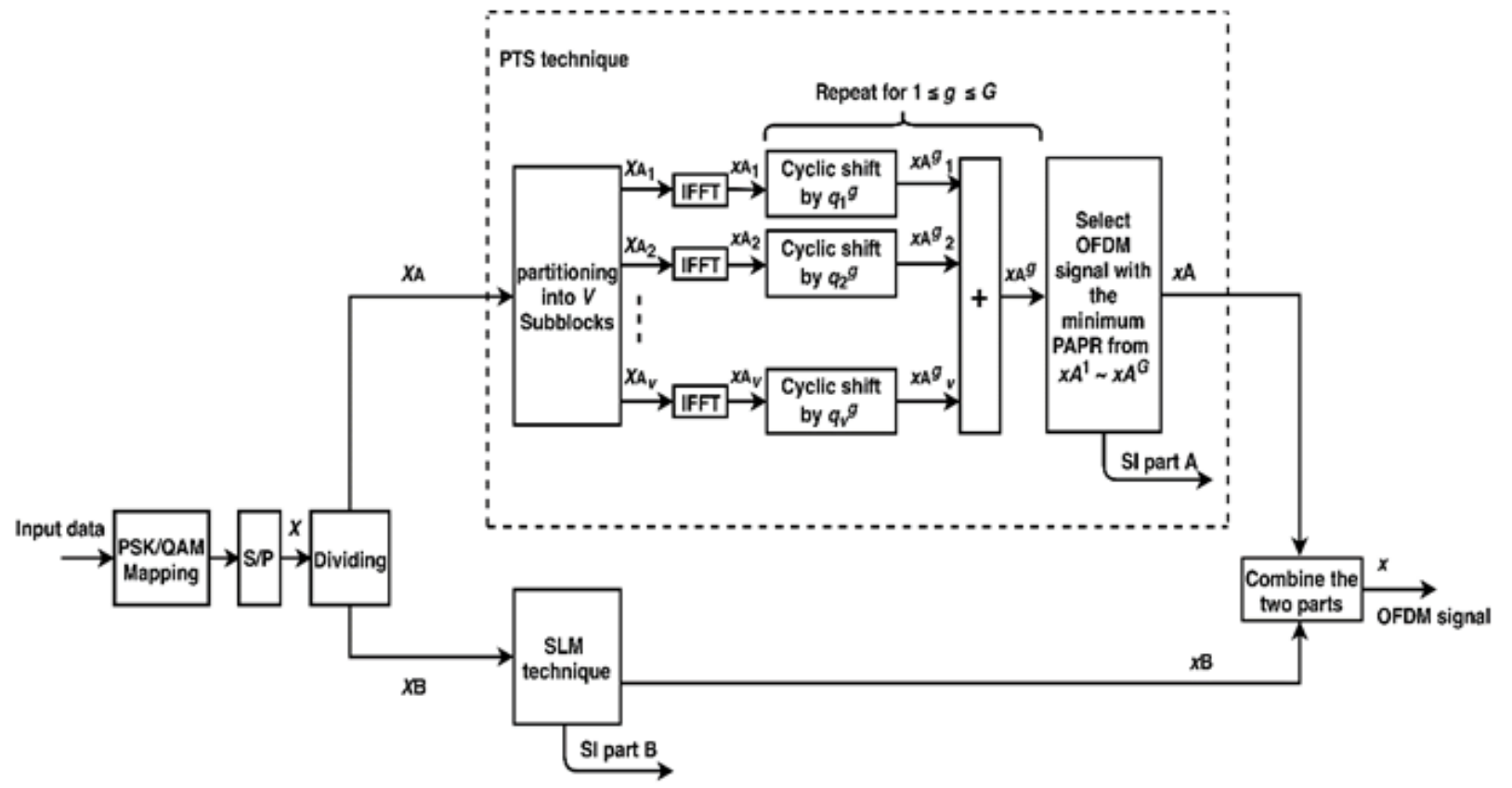

FIG 4. BLOCK DIAGRAM OF (SLM-CSS-PTS) METHOD

Thus, 


$$
X \mathrm{~A}=\left[X_{0}, X_{1}, X_{2}, \ldots, X_{(N / 2)-1}\right]
$$

, and

$$
X \mathrm{~B}=\left[X_{N / 2}, X_{(N / 2)+1}, X_{(N / 2)+2}, \ldots, X_{N-1}\right]
$$

After that, the data of part A undergoes the PTS technique, while the data of part B undergoes the SLM technique. Next, the first part employs the CSS-PTS procedure to reduce its PAPR, Where, instead of phase rotation variables, the cyclic shift technique is used to optimize the transformed subblocks to produce the optimal signal of Part A,

$$
x \mathrm{~A}=\sum_{v=1}^{V}\left\{\operatorname{circular}\left(x \mathrm{~A}_{v}^{g}\right) \mid g=1,2, \ldots, G\right\},
$$

where $(V)$ is a totally sub-block number, and $(G)$ is the total number of signals for the candidate. On the other hand, part B utilizes the SLM procedure to diminish its PAPR, where the SLM technique produces the optimum signal of part B,

$$
x \mathrm{~B}=\operatorname{IFFT}\left(X \mathrm{~B}^{u}\right) \quad, \quad u=1,2, \cdots, U .
$$

Finally, the optimal signals of both parts A and B are combined to produce the entire transmitting signal transmitted to the receiver with each part's side information,

$$
x=[x \mathrm{~A}, x \mathrm{~B}]
$$

Because both SLM and PTS techniques are employed to reduce the PAPR value, The PAPR reduction efficiency of the SLM-CSS-PTS method will be improved and the subcarriers will be more independent. Besides, the SLM-SC-PTS method's computational complexity is the sum of the complexity of SLM and PTS. Also, CSS-PTS mathematical calculations in the time domain will be complex addition and multiplication operations by using $\mathrm{H}$ shift number sets. Hence, the number of the complex addition operations ( $\mathrm{C}^{\text {SLM-CSS-PTS }}{ }_{\text {add }}$ ) and multiplication operations ( $C_{\text {mult }}^{\text {SLM-CSS-PTS }}$ ) of the SLMCSS-PTS method can be formulated as

$$
\begin{aligned}
& C_{\text {add }}^{\text {SLM-CSS-PTS }}=(U+V)\left[\frac{N}{2} \log _{2} \frac{N}{2}\right]+\left[\frac{N}{2} \times H \times(V-1)\right], \\
& C_{\text {mult }}^{\text {SLM-CSS-PTS }}=[U N]+\left[(U+V)\left(\frac{N}{4} \log _{2} \frac{N}{2}\right)\right]+ \\
& \\
& {\left[\frac{N}{2}(H+(H-1) \times(V-1))\right] }
\end{aligned}
$$

Besides, the SLM-CSS-PTS side information bit number is the sum of the SLM side information and the CSS-PTS side information,

$$
\mathrm{SI}^{\text {SLM-CSS-PTS }}=\log _{2} U+\log _{2} H .
$$




\section{SIMULATION RESULTS AND DISCUSSION}

It is suggested to use the low computational difficulty level of the SLM methodology and the superior PAPR reduction performance of the CSS-PTS approach in one system, the SLM-CSS-PTS process. In this section, the OFDM system evaluates the PAPR and BER, PSD, side details, and computational complexity outputs of SLM-CSS-PTS. In Table (1) The parameter values in the literature for testing the SLM-CSS-PTS scheme related to the other OFDM approaches.

The AWGN channel block used in an ofdm system [37] [38] [39] applies Gaussian white noise to an individual or complex input signal. This block introduces real Gaussian noise when the input signal is low, and generates a true output signal. This block introduces complex Gaussian noise when the input signal is complex, and generates a complex output signal.

TABLE 1. SIMULATION OF THE SLM-CSS-PTS PARAMETERS IN OFDM.

\begin{tabular}{l|l}
\hline \multicolumn{1}{c|}{ Simulation Parameters } & \multicolumn{1}{c}{ Values } \\
\hline$(N):$ NO. of sub-carriers & 1024,2048 \\
\hline$(M):$ NO. of the constellation & $(64)$ \\
\hline$(L):$ NO. of Oversampling & $(4)$ \\
\hline$(V):$ NO. of sub-blocks & $(4)$ \\
\hline$(W):$ NO.of phase factors & $(\{ \pm 1\},\{ \pm 1, \pm \mathrm{j}\})$ \\
\hline NO. of sub-frames & $(1000)$ \\
\hline$(r):$ Roll-off factor & $(0.6)$ \\
\hline Modulation type & $M$-QAM \\
\hline Determine value $(\alpha)-$ Xiaoqiang method & $(0.5)$ \\
\hline Number of phase rotation vectors (PRV) - SLM method & $(24)$ \\
\hline Channel model & AWGN \\
\hline
\end{tabular}

\section{A. PAPR 'S RESULTS FOR SLM-CSS-PTS.}

The modeling takes place in the OFDM system whenever the number of the subcarrier is 2048 and the constellation order $M=64$. The SLM-CSS-PTS algorithm performs other methods concerning reductions in PAPR performance. As seen in Fig.5, when CCDF OF SLM-CSS-PTS, PR-PTS, SLM, K. Singh's method, Xiaoqiang 's method, Tiwari's method, and OFDM's original value. Besides, the SLM-CSS-PTS BER output is close to that of the original OFDM signal, as seen in Fig.6. This is because the SLM-CSS-PTS procedure is a form of MSR methodology, where the SLM-CSS-PTS approach has no signal distortions. Also we can see comparison of the number of complex multiplications for suggested method and other methods in Fig.7. 


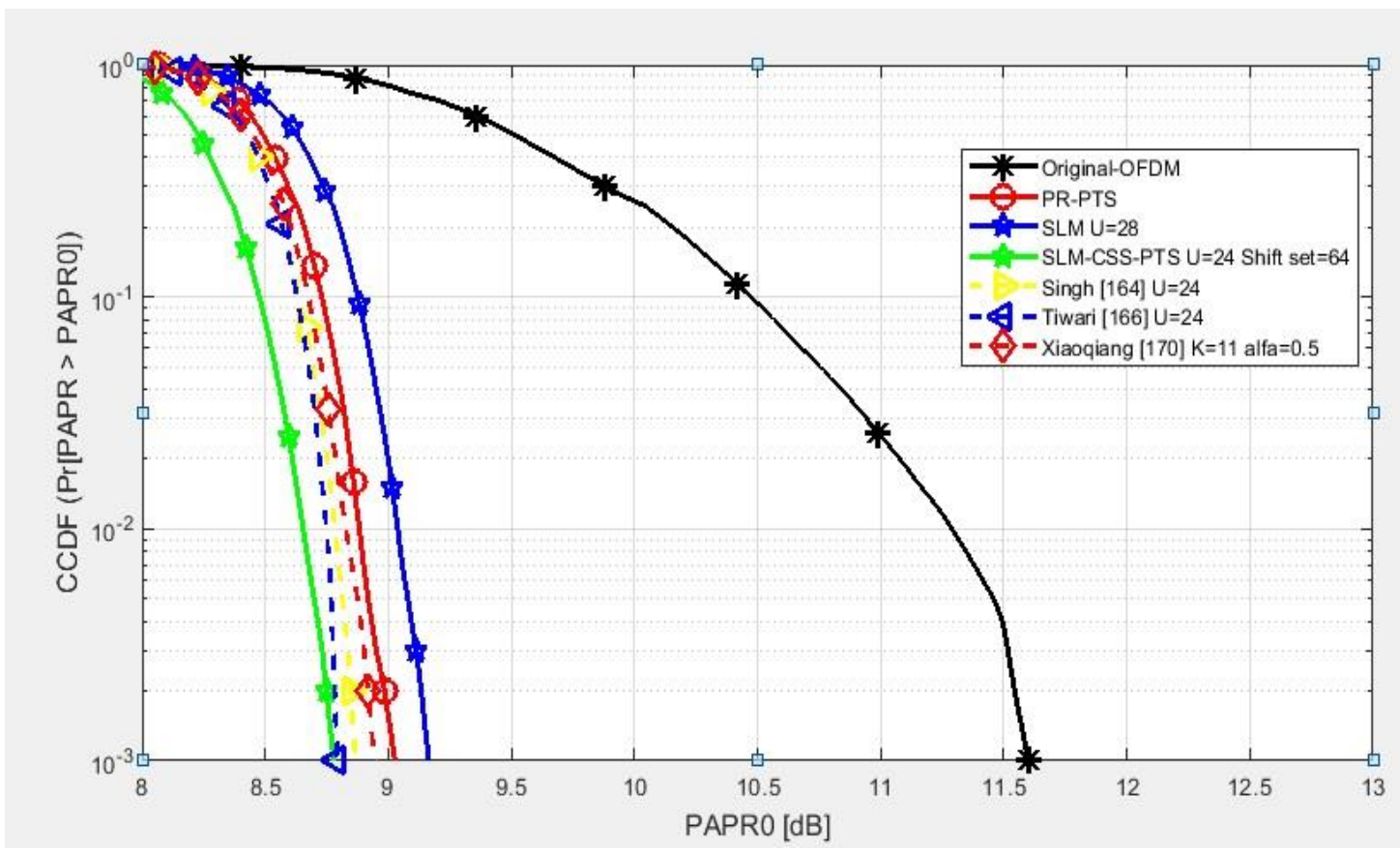

FIG 5. CCDF COMPARITION PAPR FOR THE PROPOSED METHOD WITH THE OTHER METHODS, NO. OF SUBCARRIER $=2048, \mathrm{M}=64$.

\section{B. Computational Complexity of SLM-CSS-PTS Compared With Other methods}

The difficulty of the calculation here reflects the sum of complicated contributions in the frequency and time domains and complicated multiplications. In this section, the SLM-CSS-PTS system's mathematical estimation is conducted and compared with PR-PTS and some similar methods discussed in the literature. Table (2) details complex add-on and multiplication equations in the sources for PRPTS, SLM-CSS-PTS, and several of the integrated processes.

Table (3) shows the number of complex adding and multiplication processes for the various methods in Table (2). The results indicate that SLM-CSS-PTS is the lowest computational complexity among methods combined.

Table (4) offers a comparison of the computational complexity reduction ratio (CCRR) results between the SLM-CSS-PTS approach and the other relevant approaches. The numerical complexity, degree of the SLM-CSS-PTS approach is higher than other combined techniques. For example, when $N=1024$, the complex addition operations of SLM-CSS-PTS have been reduced by $4.31 \%, 52.15 \%$, $52.96 \%, 53.94 \%, 0 \%, 31.05 \%$, and 31.05\% compared with PR-PTS, K. Singh and Tiwari, respectively. Besides, the complex multiplication operations of SLM-CSS-PTS have been reduced by $37.2 \%$, $68.69 \%, 57.97 \%, 58.38 \%, 13.56 \%, 45.81 \%$, and $45.81 \%$ compared with the PR-PTS, K. Singh and Tiwari, respectively. Consequently, in contrast to other hybrid methods, the SLM-CSS-PTS solution is known to be an efficient tool for optimizing PAPR reduction performance at low computational complexity. 
TABLE 2. PR-PTS COMPLEXITY EQUATION, SLM-CSS-PTS, AND SOME OF THE METHODS PREVIOUSLY COMBINED IN THE LITERATURE.

\begin{tabular}{c|c|c}
\hline Technique & $\boldsymbol{C}_{\text {mult }}$ & $\boldsymbol{C}_{\text {add }}$ \\
\hline SLM & {$[2 U N]+\left[\frac{U N}{2} \log _{2} N\right]$} & {$\left[U N \log _{2} N\right]$} \\
\hline PR-PTS & {$\left[\frac{V N}{2} \log _{2} N\right]+\left[W^{V-1} \times N \times(V+1)\right]$} & {$\left[V N \log _{2} N\right]+\left[W^{V-1} \times N \times(V-1)\right]$} \\
\hline SLM-CSS-PTS & {$[U N]+\left[(U+V)\left(\frac{N}{4} \log _{2} \frac{N}{2}\right)\right]+$} & $(U+V)\left[\frac{N}{2} \log _{2} \frac{N}{2}\right]+$ \\
Tiwari[19] & {$\left[\frac{N}{2}(H+(H-1) \times(V-1))\right]$} & {$\left[\frac{N}{2} \times H \times(V-1)\right]$} \\
\hline K. Singh[17] & {$[2 U N]+\left[(U+V)\left(\frac{N}{2} \log _{2} N\right)\right]+$} & {$\left[(U+V+1)\left(\frac{N}{2} \log _{2} N\right)\right]+$} \\
{$\left[W^{V-1} \times N \times(V+1)\right]$} & {$[U+V+1)\left[N \log _{2} N\right]+$} \\
& {$[K N]+\left[(K+V)\left(\frac{N}{2} \log _{2} N\right)\right]+$} & {$\left[W^{V-1} \times N \times(V-1)\right]$} \\
& {$\left[W^{V-1} \times N \times(V+1)\right]$} & {$\left[W^{V-1} \times N \times(V-1)\right]$} \\
\hline
\end{tabular}

TABLE 3. SLM-CSS-PTS COMPUTATIONAL COMPLEXITY, AND THE VARIOUS COMBINED LITERATURE METHODS.

\begin{tabular}{|c|c|c|c|c|c|c|c|c|c|c|c|c|}
\hline \multirow{2}{*}{$\boldsymbol{N}$} & \multicolumn{2}{|c|}{ PR-PTS } & \multicolumn{2}{|c|}{ SLM } & \multicolumn{2}{|c|}{ K. Singh [17\} } & \multicolumn{2}{|c|}{ Tiwari [19] } & \multicolumn{2}{|c|}{ Xiaoqiang [20] } & \multicolumn{2}{|c|}{ SLM-CSS-PTS } \\
\hline & $\left(\boldsymbol{C}_{\text {add }}\right)$ & $\left(C_{\text {mult }}\right)$ & $\left(C_{\text {add }}\right)$ & $\left(C_{\text {mult }}\right)$ & $\left(C_{\text {add }}\right)$ & $\left(C_{\text {mult }}\right)$ & $\left(\boldsymbol{C}_{\text {add }}\right)$ & $\left(C_{\text {mult }}\right)$ & $\left(C_{\text {add }}\right)$ & $\left(C_{\text {mult }}\right)$ & $\left(C_{\text {add }}\right)$ & $\left(C_{\text {mult }}\right)$ \\
\hline 64 & 13824 & 21248 & 16 & 7680 & 23040 & 28928 & 23424 & 29120 & 15744 & 22528 & 10624 & 11872 \\
\hline 1024 & 237568 & 348160 & 245760 & 172032 & 483328 & 520192 & 493 & 525 & 329 & 403456 & 227328 & 218624 \\
\hline 2048 & 3328 & 700416 & 0672 & 368640 & 1024000 & 1069056 & 1046528 & 1080320 & 708608 & 833536 & 483328 & 451584 \\
\hline
\end{tabular}

Table 4. CCRR BETWEEN THE SLM-CSS-PTS APPROACH AND SOME OF THE COMBINED APPROACHES IN THE LITERATURE.

\begin{tabular}{|c|c|c|c|c|c|c|c|c|}
\hline \multirow{3}{*}{$\mathrm{N}$} & \multicolumn{2}{|c|}{$\begin{array}{c}\text { CCRR SLM-CSS- } \\
\text { PTS \& PR-PTS \% }\end{array}$} & \multicolumn{2}{c|}{$\begin{array}{c}\text { CCRR SLM-CSS- } \\
\text { PTS \& Pushkarev \% }\end{array}$} & \multicolumn{2}{c|}{$\begin{array}{c}\text { CCRR SLM-CSS- } \\
\text { PTS \& K. Singh \% }\end{array}$} & \multicolumn{2}{c|}{$\begin{array}{c}\text { CCRR SLM-CSS- } \\
\text { PTS \& Tiwari \% }\end{array}$} \\
\cline { 2 - 9 } & $\left(\mathrm{CCRR}^{+}\right)$ & $\left(\mathrm{CCRR}^{\times}\right)$ & $\left(\mathrm{CCRR}^{+}\right)$ & $\left(\mathrm{CCRR}^{\times}\right)$ & $\left(\mathrm{CCRR}^{+}\right)$ & $\left(\mathrm{CCRR}^{\times}\right)$ & $\left(\mathrm{CCRR}^{+}\right)$ & $\left(\mathrm{CCRR}^{\times}\right)$ \\
\hline 64 & 23.14 & 44.12 & 61.57 & 72.14 & 53.88 & 58.96 & 54.64 & 59.23 \\
\hline 1024 & 4.31 & 37.2 & 52.15 & 68.69 & 52.96 & 57.97 & 53.94 & 58.38 \\
\hline 2048 & 0 & 35.52 & 50 & 67.85 & 52.8 & 57.75 & 53.81 & 58.19 \\
\hline
\end{tabular}




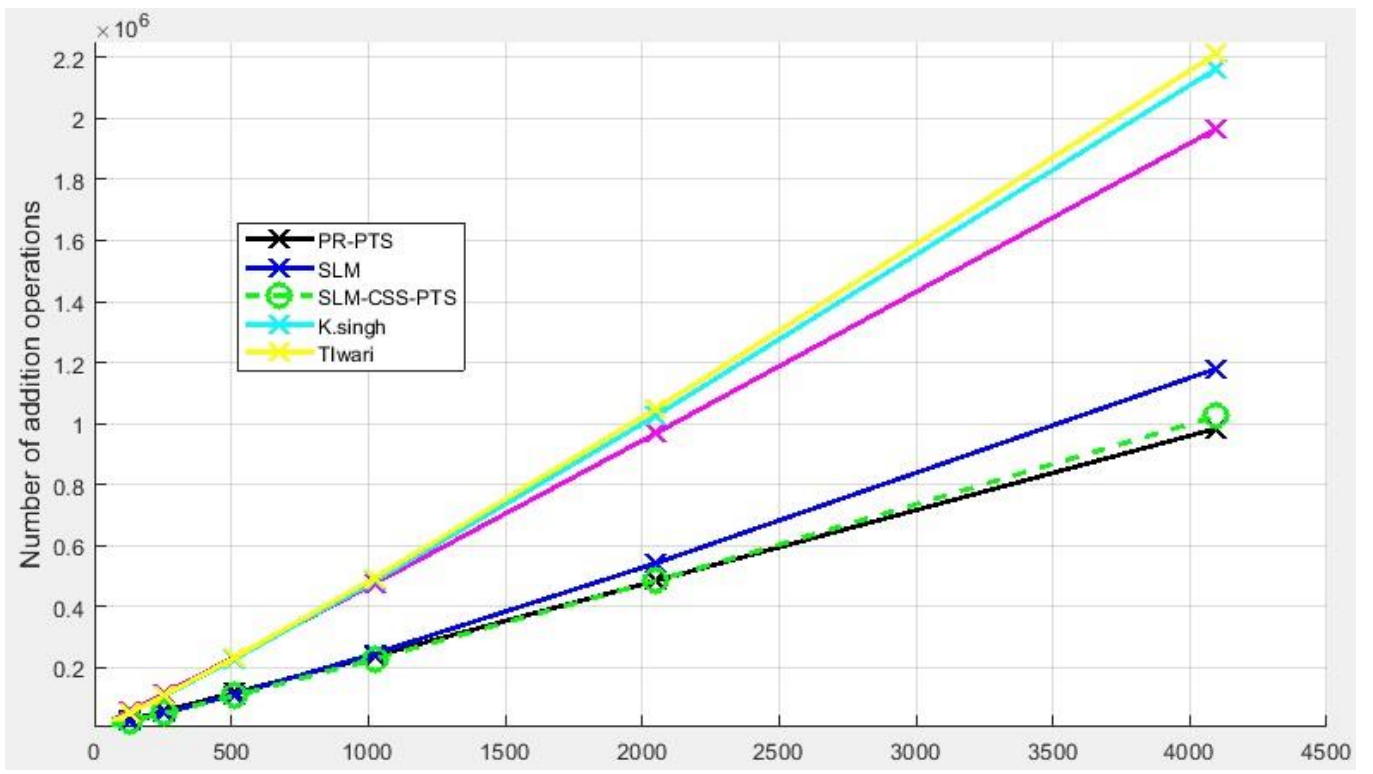

FIG 6. COMPARIDION OF COMPLEX ADDITION NUMBERS FOR DIFFERENT METHODS IN (TABLE 3).

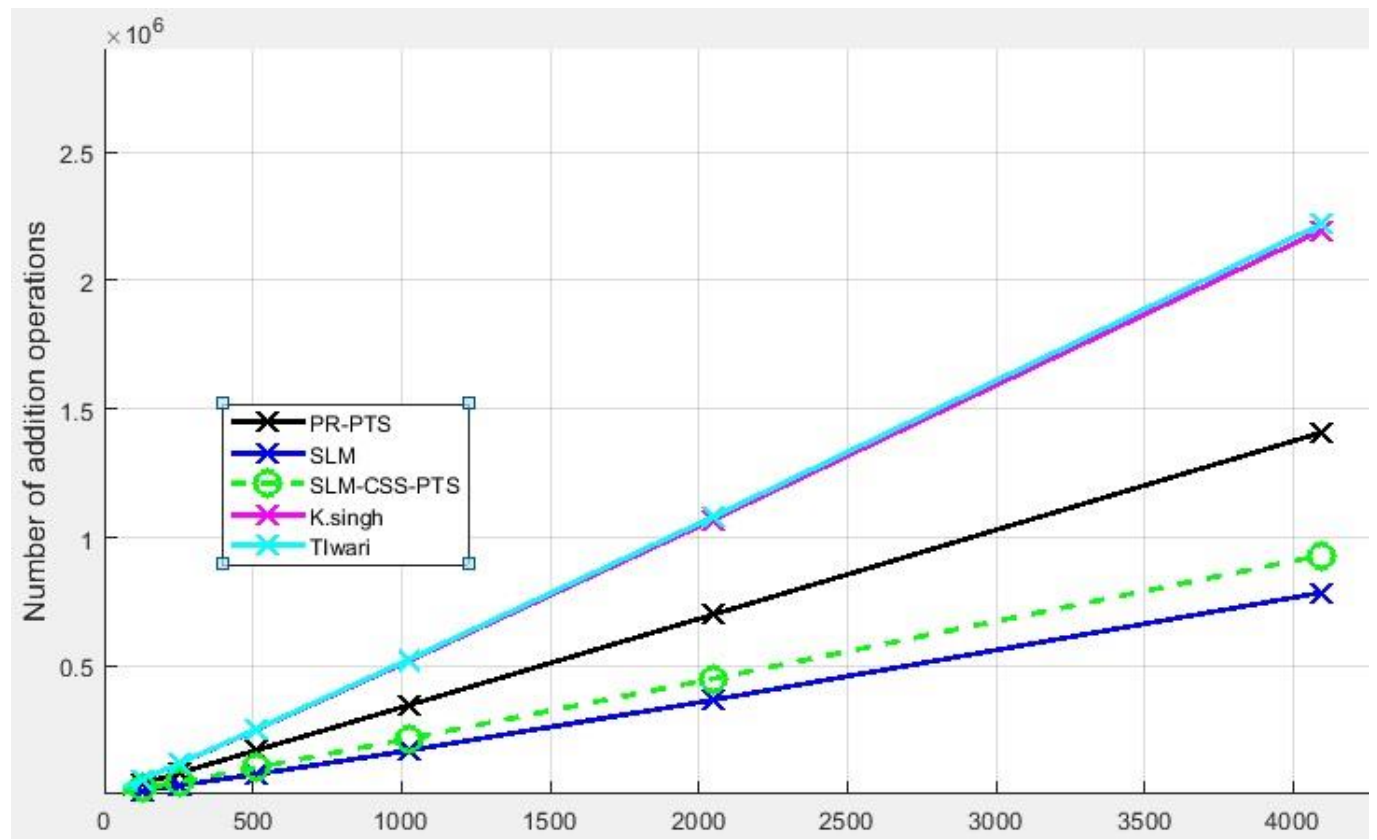

FIG.7. COMPARIDION OF THE NUMBER OF COMPLEX MULTIPLICATIONS FOR DIFFERENT ALGORITHMS IN (TABLE3).

Table 5, on the other hand, lists the side information bits needed for the various methods in this paragraph, where the SI bits of the SLM-CSS-PTS scheme can be calculated by (16). 11 bits for the method of K. Singh, the method of Tiwari 11 bits. The SLM-CSS-PTS thus have more side-information bits than the PR-PTS method, given that the suggested method is better than PR-PTS in terms of PAPR output reduction and computational complexity level.

In comparison, the suggested approach has a smaller or an equivalent number of side data bits compared to the other blended methods. 
Table (5).SIDE INFORMATION ABOUT SLM-CSS-PTS AND SOME OF THE LITERATURE'S COMBINED METHODS.

\begin{tabular}{|c|c|c|c|c|c|}
\hline \multicolumn{7}{|c|}{$V=4, W=4, H=64, U=24$} \\
\hline Method & PR-PTS & SLM & SLM-CSS-PTS & K. Singh & Tiwari \\
\hline SI & 6 & 5 & 11 & 11 & 11 \\
\hline
\end{tabular}

\section{CONCLUSION}

This research developed a hybrid multiple signal representation (MSR) approaches to reduce the high PAPR sequence in OFDM with low transmitter signal complexity. The suggested hybrid solution incorporates the SLM framework and the CSS-PTS solution in tandem, the SLM methodology has strong low-complexity output reduction PAPR and the CSS-PTS approach has excellent mediumcomplexity PAPR cost reduction. Simulations and numerical calculations were carried out to evaluate the hybrid method proposed for the reduction of PAPR power, computational complexity, and side details. It's been shown that the hybrid proposed method could enhance PAPR reduction performance, greater than the PTS method, and the current hybrid approach without deterioration. Meanwhile, the suggested hybrid approach is lower in computational complexity than the PTS method and presents hybrid methods. In comparison, the number of side information bits of the new system is less than currently in use hybrid methods. Accordingly, it is concluded that the proposed SLM-CSS-PTS approach is ideal to reduce the high PAPR pattern in the low computational complexity OFDM system.

\section{REFERNCES}

[1] Jiao, Long, "Physical layer key generation in 5G wireless networks." IEEE Wireless Communications 26.5 ,2019, pp. $48-54$.

[2] Ibrahim, A. N., and M. F. L. Abdullah. "The potential of FBMC over OFDM for the future 5G mobile communication technology." AIP Conference Proceedings. Vol. 1883. No. 1. AIP Publishing LLC, 2017.pp.20001.

[3] Jawhar, Yasir Amer, et al. "A review of partial transmit sequence for PAPR reduction in the OFDM systems." IEEE Access 7 ,2019, pp.18021-18041.

[4] Shu'aibu, D. S., S. K. Syed-Yusof, and N. Fisal. "Partition-Based Bandwidth Management for Mobile WiMAX IEEE 802. 16 e." International Review on Computers and Software 5.4 ,2010, pp. 445-452.

[5] Yi, Wang, and Harry Leib. "OFDM symbol detection integrated with channel multipath gains estimation for doublyselective fading channels." Physical communication 22 ,2017. pp.19-31.

[6] Chen, Huixiang, "Understanding the characteristics of mobile augmented reality applications." IEEE International Symposium on Performance Analysis of Systems and Software (ISPASS). IEEE, 2018, pp.128-138.

[7] Gokceli, Selahattin, and Gunes Zeynep KARABULUT KURT. "Superposition coded orthogonal frequency division multiplexing (SC-OFDM) system." U.S. Patent No. 10,411,934. 10 Sep. 2019.

[8] Taher, Montadar Abas. "NON-LINEARITY DISTORTION MITIGATION OF DOWNLINK-LTE SYSTEM USING MODIFIED AMPLITUDE CLIPPING AND FREQUENCY DOMAIN RANDOMIZATION: Communication." Diyala Journal of Engineering Sciences 8.4, 2015, pp. 316-617.

[9] Zou, Wuhua, "Modified constellation reshaping method for PAPR reduction of PDM CO-OFDM based on a SLM algorithm." Applied optics 58.7 ,2019, pp.1800-1807.

[10] Ali, Nazar, "Analysis of improved $\mu$-law companding technique for OFDM systems." IEEE Transactions on Consumer Electronics 63.2, 2017, pp.126-134.

[11] Gabriel, Frank, "Multipath communication with finite sliding window network coding for ultra-reliability and low latency." 2018 IEEE International Conference on Communications Workshops (ICC Workshops). IEEE, 2018. pp.16

[12] Taher, M. A."Sliding the SLM-technique to reduce the non-linear distortion in OFDM systems." Elektronika ir Elektrotechnika 19.5, 2013,pp.103-111.

[13] Jawhar, Yasir Amer, "New low- complexity segmentation scheme for the partial transmit sequence technique for reducing the high PAPR value in OFDM systems." ETRI Journal 40.6,2018, pp.699-713.

[14] Joo, Hyun-Seung, "New PTS schemes for PAPR reduction of OFDM signals without side information." IEEE Transactions on Broadcasting 63.3.2017, pp.562-570. 
[15] Taher, Montadar Abas, "Post-IFFT-modified selected mapping to reduce the PAPR of an OFDM system." Circuits, Systems, and Signal Processing 34.2.2015, pp. 535-555.

[16] Pushkarev, Pavel A., et al. "A study on the PAR reduction by hybrid algorithm based on the PTS and SLM techniques." The 57th IEEE Semiannual Vehicular Technology Conference, 2003. VTC 2003-Spring.. Vol. 2. IEEE, 2003. pp.1263-1267.

[17] Singh, Kamal, Manoranjan Rai Bharti, and Sudhanshu Jamwal. "A modified PAPR reduction scheme based on SLM and PTS techniques." 2012 IEEE International Conference on Signal Processing, Computing and Control. IEEE, 2012. pp. 1-6.

[18] Mohammad, A. S., Abdel Halim Zekry, and Fatama Newagy. "A combined PTS-SLM scheme for PAPR reduction in multicarrier systems." 2013 IEEE Global High Tech Congress on Electronics. IEEE, 2013. pp.146-150.

[19] Tiwari, Harshita, Rakesh Roshan, and Rajat Kumar Singh. "PAPR reduction in MIMO-OFDM using combined methodology of selected mapping (SLM) and partial transmit sequence (PTS)." 2014 9th International Conference on Industrial and Information Systems (ICIIS). IEEE, 2014. pp.1-5.

[20] Xiaoqiang, Qiao, and Liang Tao Nanjing. "Comparison analysis of multiple signal representation methods for PAPR reduction in OFDM systems." Proceedings of 2013 3rd International Conference on Computer Science and Network Technology. IEEE, 2013. pp. 850-853.

[21] Satyavathi, Kakara, and B. Rama Rao. "Modified phase sequence in hybrid PTS scheme for PAPR reduction in OFDM systems." Innovations in electronics and communication engineering. Springer, Singapore, 2019. 327-333.

[22] Wang, Han. "A hybrid PAPR reduction method based on SLM and multi-data block PTS for FBMC/OQAM systems." Information 9.10 , 2018, pp.246.

[23] Kim, Kee-Hoon. "On the shift value set of cyclic shifted sequences for PAPR reduction in OFDM systems." IEEE Transactions on broadcasting 62.2. 2016, pp.496-500.

[24] Yasir Amer; abdulhasan, Raed A.; RAMLI, Khairun Nidzam. "A new hybrid sub-block partition scheme of PTS technique for reduction PAPR performance in OFDM system". ARPN journal of engineering and applied sciences, 11.6. 2016, pp. 3904-3910.

[25] Jawhar, Yasir Amer, "New low- complexity segmentation scheme for the partial transmit sequence technique for reducing the high PAPR value in OFDM systems." ETRI Journal 40.6. 2018, pp.699-713.

[26] Al-Jawhar, Yasir Amer, et al. "A new partitioning scheme for PTS technique to improve the PAPR performance in OFDM systems." International Journal of Engineering and Technology Innovation 8.3. 2018, pp.217.

[27] Jawhar, Y.. "A low PAPR performance with new segmentation schemes of partial transmit sequence for OFDM systems." IJAAS International Journal of Advanced and Applied Sciences 4.4. 2017, pp.14-21.

[28] Al-Jawhar, Yasir Amer, et al. "Zero-padding techniques in ofdm systems." International Journal on Electrical Engineering and Informatics 10.4 . 2018, pp.704-725.

[29] Yu, Pingyuan, and Shubo Jin. "A low complexity tone reservation scheme based on time-domain kernel matrix for PAPR reduction in OFDM systems." IEEE transactions on broadcasting 61.4.2015. pp.710-716.

[30] Muller, Stefan H., and Johannes B. Huber. "A novel peak power reduction scheme for OFDM." Proceedings of 8th International Symposium on Personal, Indoor and Mobile Radio Communications-PIMRC'97. Vol. 3. IEEE, 1997. pp. 1090-1094.

[31] Bauml, Robert W., Robert FH Fischer, and Johannes B. Huber. "Reducing the peak-to-average power ratio of multicarrier modulation by selected mapping." Electronics letters 32.22.1996. pp. 2056-2057.

[32] Kim, Kee-Hoon. "On the shift value set of cyclic shifted sequences for PAPR reduction in OFDM systems." IEEE Transactions on broadcasting 62.2 .2016. pp. 496-500.

[33] Joo, Hyun-Seung,"A new blind SLM scheme with low decoding complexity for OFDM systems." IEEE transactions on broadcasting 58.4. 2012. pp. 669-676.

[34] Hu, Wei-Wen, and Da-Huei Lee. "PAPR reduction for visible light communication systems without side information." IEEE Photonics Journal 9.3 .2017. pp.1-11.

[35] Yang, Lin, et al. "PAPR reduction using low complexity PTS to construct of OFDM signals without side information." IEEE Transactions on Broadcasting 57.2 .2011. pp.284-290.

[36] Long, Ken, Yue Fu, and Yi Wang. "The contradiction between channel estimation and PAPR performance in cyclic shift PTS." 2012 5th International Conference on BioMedical Engineering and Informatics. IEEE, 2012. pp.15251528.

[37] Manhas, Pratima, and M. K. Soni. "Implementation of OFDM System Using Image Input for AWGN Channel." Innovations in Computer Science and Engineering. Springer, Singapore, 2020. pp.7-13. 
[38] Dharavathu, Krishna, and Anuradha Mosa. "Secure image transmission through crypto- OFDM system using Rubik's cube algorithm over an AWGN channel." International Journal of Communication Systems 33.8 .2020. pp.e4369.

[39] Jiang, Tao, Cai Li, and Chunxing Ni. "Effect of PAPR reduction on spectrum and energy efficiencies in OFDM systems with class-A HPA over AWGN channel." IEEE Transactions on Broadcasting 59.3 .2013. pp.513-519. 